\title{
Bias in Methicillin Resistance of Staphylococcus aureus
}

\author{
Ramezan Ali Ataee ${ }^{1, *}$ \\ ${ }^{1}$ Department of Medical Microbiology, Faculty of Medicine, Baqiyatallah University of Medical Sciences, Tehran, IR Iran \\ "Corresponding author: Ramezan Ali Ataee, Department of Medical Microbiology, Baqiyatallah University of Medical Sciences, Mollasadra Ave, P. O. Box: 193955487, Tehran, IR \\ Iran. Tel: +98-9122190418, Fax: +98-26127258, E-mail: ataee@bmsu.ac.irataee216@gmail.com
}

Received 2014 December 10; Revised 2015 February 28; Accepted 2015 June 16.

Keywords: Bias, Methicillin Resistance, Polymerase Chain Reaction, Staphylococcus aureus

\section{Dear Editor}

Recently, several published papers aiming to isolate and characterize methicillin resistant Staphylococcus aureus (MRSA) have had major bias. For example, an article titled "Microbial Susceptibility and Plasmid Profiles of Methicillin-Resistant Staphylococcus aureus and Methicillin-Susceptible S. aureus" by Shahkarami et al. (1). Even though, the authors have spent respectable effort to report the degree of methicillin resistance of $S$. aureus, the unrealistic results of the study inspired us to write this letter. The mentioned paper had the following fundamental scientific and technical problems: Repeated words in the title were predominant; Staphylococcus aureus and Methicillin were repeated. In the methods section, it was mentioned that between November and January of 2013, in about eleven months, 500 outpatients or hospitalized patients were checked, and in 106 strains of S. aureus were detected. However, the inclusion and exclusion criteria for selecting patients were not provided. Also, the most of the report are related to the obtained results of the laboratory routines daily work and was not specified. In addition, in the methods section, and also in Figure 2 of the article, amoxicillin was not noted, while in the results and discussion it was mentioned. It may be that the authors, have mistaken it with ampicillin.

The respectable authors had examined 11 antibiotics, yet the selection criteria for these antibiotics are not clear. Selection of relevant antimicrobial agents should be based on specific criteria. These criteria should be extensively considered when antimicrobial agents are selected, to avoid cluttering reports with superfluous information and time consuming and costly procedures, and to minimize the risk of confusing physicians (2). The authors did not explain why in addition to the penicillin disk, ampicillin was also chosen. Similarly, the choice of nalidixic acid disk along with ciprofloxacin was unclear. The investigators did not notice that nalidixic acid is the main composition of the selective media for isolation and differentiation of $S$. aureus (2). On the basis of the available knowledge, the an- tibiotic disks are usually loaded with $10 \mu \mathrm{g}$ of penicillin and $5 \mu \mathrm{g}$ of ciprofloxacin. It is not clear, why the respected authors selected penicillin and ciprofloxacin disks of $5 \mu \mathrm{g}$ and $30 \mu \mathrm{g}$, respectively. Thus, wrong results in Figure 2 of the article are shown. For example 100\% methicillin resistant strains were resistant to penicillin, which is not in agreement with other studies $(3,4)$.

In addition, the respected authors mentioned that the antibiotic disks were from Iranian PATAN TEB Co., yet the disk information's such as: serial number, production expiration date, the period of validity and storage conditions were not specified. Furthermore, the respectable scholars in section 3.2 of the methods stated that all methicillin resistant strains had been evaluated with E test strips of oxacillin and vancomycin, which were from Iranian PATAN TEB Co., but the question is whether this company really produces E test strips. It would be great if the respected scholars reported their exact specifications. The authors did not consider the results of other national published researches. While, the standard protocols for MRSA isolation is available (5).

The respected researchers, with the use of primer pairs, amplified a fragment of $533 \mathrm{bp}$, as the mecA gene, yet did not give any reason for its approval. It would have been better to use sequencing and/or enzymatic digestion to confirm the Polymerase Chain Reaction (PCR) products. Based on the previuse published paper which, they reported that the mecA gene encoding a $78-\mathrm{kDa}$ protein, is named as PBP2a; it would have been worthy to explain why a primer pair has been chosen that amplifies a fragment of 533-bp. Figure 1 of the article lacked positive and negative controls. Also it was not clear which method confirmed the PCR products. The authors used S. aureus ATCC 29213 and S. epidermidis ATCC 35984 as positive and negative controls, respectively. Based on the ATCC catalogue site, the strain 29213 is a quality control strain for susceptible disk testing. While, the strain 35984 is a polysaccharide adhesion producer and it is not clear why the authors used of this strain. This study needed positive and negative controls for mecA 
gene detection, as found in the study of Erami et al. (6).

According to table 2 of the article, methicillin minimum inhibitory concentrations (MICs) for three of the strains was equal to $128 \mu \mathrm{g} / \mathrm{mL}$ while for four strains this was equal to $256 \mu \mathrm{g} / \mathrm{mL}$. Presenting such large methicillin MIC requires confirmed evidence. It would be necessary for the respected scholars to show the corresponding E test strip plate Figures to support their data. The respected authors in the results section had written the following statement: "The frequency of $S$. aureus isolated from different sources is shown in Table 3 of the mentioned article", while Table 3 of the article presented the design of the distribution of the plasmid profile. It would be worthy for the respected authors to provide some evidence of plasmid profiles and determine the purpose of specifying the plasmid profiles such as the study of Champion et al. (7).

The use of irrelevant reference, for example reference number 17 is noteworthy. In the fourth line of the results section the authors had stated "a total of 67 isolates (63.20 $\%$ ) were selected as MRSA and were analyzed", while in the beginning of the last paragraph of the results section this phrase was written, "In PCR, mecA gene was detected in 62 of the MRSA isolates" (Figure 1 of the mentioned article), however, it is unclear by which method the authors confirmed that $92.53 \%$ of the isolates were MRSA. These data were not scientifically justified and explained. If the authors intended to show that $92.53 \%$ of the strains were MRSA carrying the mecA, they should have represented scientific explanation for the other $7.5 \%$ of the strains that lacked mecA but were resistant to methicillin. Nevertheless, from an antibiotic resistant standpoint, MRSA is important and should be considered crucial for prevention and treatment of S. aureus. Therefore, studies in this field must focus on the advantages, disadvantages, accuracy and reliability of the research. Such evaluations will not only enhance medical team members and researchers' understanding of the existing facts but also lay the ground for future research.

\section{Acknowledgments}

The author would like to thank Professor Ali Mehrabi Tavana for encouraging him to write this letter.

\section{References}

1. Shahkarami F, Rashki A, Rashki Ghalehnoo Z. Microbial Susceptibility and Plasmid Profiles of Methicillin-Resistant Staphylococcus aureus and Methicillin-Susceptible S. aureus. Jundishapur J Microbiol. 2014;7(7):ee16984. doi: 10.5812/jjm.16984. [PubMed: 25368805].

2. Patricia MT. Baily \& Scott's DIAGNOSTIC MICROBIOLOGY, Thirteenth edition, MOSBY ELSEVIER. South Dakota State University. 2014:168-90.

3. Ataee RA, Irannejad L, Esmaeili D, Mehrabi TA, Salesi M. Antibiotic Resistance profiles Determine of bacteria isolated from the operating room in a Hospital in 2013 Sumer. The 23rd Iranian Congress on Infectious Disease and Tropical Medicine 12-16 Jan. 2015. p. 297.

4. Dibah S, Arzanlou M, Jannati E, Shapouri R. Prevalence and antimicrobial resistance pattern of methicillin resistant Staphylococcus aureus (MRSA) strains isolated from clinical specimens in Ardabil, Iran. Iran J Microbiol. 2014;6(3):163-8. [PubMed: 25870749].

5. Motevasel M, Okhovat MA, Zomorodian K, Farshad S. A Study of the Effect of Zataria multiflora Extract on Methicillin Resistant Staphylococcus aureus. Jundishapur J Microbiol. 2013;6(5):e5453. doi: 10.5812/jjm.5453.

6. Erami M, Soltani B, Taghavi Ardakani A, Moravveji A, Haji Rezaei M, Soltani S, et al. Nasal Carriage and Resistance Pattern of Multidrug Resistant Staphylococcus aureus Among Healthy Children in Kashan, Iran. Iran Red Crescent Med J. 2014;16(9):ee21346. doi: 10.5812/ircmj.21346. [PubMed: 25593734].

7. Champion AE, Goodwin TA, Brolinson PG, Werre SR, Prater MR, Inzana TJ. Prevalence and characterization of methicillin-resistant Staphylococcus aureus isolates from healthy university student athletes. Ann Clin Microbiol Antimicrob. 2014;13:33. doi: 10.1186/s12941-014-0033-5. [PubMed: 25085442]. 